\title{
EDITORIAL
}

\section{Tumor marker and hepatic neoplasms}

Adagmar Andriolo

Beside metabolic diseases, neoplastic diseases have become largely responsible for the morbidity and mortality in the XXI century. Some cancers have a strong relationship with predisposing, environmental or behavioral factors, enabling the adoption of prophylactic measures aiming to reduce its incidence. Others are caused by genetic mutations, offering lower odds of preventive actions. Regardless of the triggering event, a feature common to virtually all cancers is that early diagnosis is closely related to the improvement of patient prognosis. For this reason, the demand for sufficiently sensitive and specific diagnostic capabilities is highly desirable. Another focus of current studies on cancer relates to the ability of some biochemical markers may provide information on the nature of the lesion, benign or not.

In relation to primary liver cancers, according to the consolidated data on cancer mortality in Brazil, provided by the National Cancer Institute, cancer of the liver and biliary tract was responsible for 8,100 deaths, 4,556 men and 3,544 women in 2011 (2). Primary neoplasms of the liver include hepatocellular carcinoma, cholangiocarcinoma, angiosarcoma and hepatoblastoma.

Hepatocellular carcinoma is the most common primary liver tumors, occurring in over $80 \%$ of cases. Its incidence rate in Brazil ranges from about 1-9 per 100 thousand inhabitants. In Southeast Asia, Japan and South Africa the incidence of this cancer is particularly high. Alpha-fetoprotein is a serum tumor marker that is elevated in about 75\% to $90 \%$ of patients. Cholangiocarcinoma affects the intrahepatic bile ducts and is responsible for $5 \%$ of cases of primary liver tumor. Usually occurs between the $6^{\text {th }}$ and $7^{\text {th }}$ decades of life. The angiosarcoma originates in the blood vessels in response to the carcinogenic effect of some chemicals and hepatoblastoma develops early in life.

In this edition of JBPML, you will find a paper entitled "The mucins and NCAM (CD56) in intrahepatic colangiocarcinogênese" ${ }^{(1)}$, in which the participation of different types of mucins and some adhesion molecules (NCAM) expressed in bile ducts is discussed as differential markers between reactive and dysplastic processes ${ }^{(3)}$. In addition, a particular type of mucin, MUC1 was associated with the presence of metastases, loss of differentiation and more invasive behavior of the tumor, worse prognosis, which would be reflected in reduced survival ${ }^{(4)}$. Is still a very controversial issue and the literature provides us with conflicting results, in part by the concept and discrimination between reactive and dysplastic processes and varying degrees of tumor differentiation studied.

Certainly, these differences will bring more colorful and more importance to the reading of this number of JBPML. Have an enjoyable reading.

Clinical pathologist; professor of Laboratory Medicine, Escola Paulista de Medicina (UNIFESP); editor in chief of the Brazilian Journal of Pathology and Laboratory Medicine. 


\section{REFERENCES}

1. ESPERANÇA, A. B. T. et al. Mucins and NCAM (CD56) in intrahepatic cholangiocarcinogenesis. JBPML, v. 50, n. 3, p. 216-20, 2014.

2. INCA. Instituto Nacional do Câncer. Available at: <http://www2.inca.gov.br/wps/wcm/connect/tiposdecancer/site/home/figado> Accessed on: Jun. 16, 2014.

3. MALL, A. S. et al. The expression of MUC in cholangiocarcinoma. Pathol Res Pract, v. 206, n.12, p. 805-9, 2010.

4. PARK, S. Y. et al. Expression of MUC1, MUC2, MUC5AC and MUC6 in cholangiocarcinoma: prognostic impact. Oncol Rep, v. 22, n. 3 , p. 649-57, 2009 . 